\title{
Absolute Calibration of the National Bureau of Standards Photoneutron Standard: II. Absorption in Manganese Sulfate
}

\author{
James De Juren ${ }^{1}$ and Jack Chin
}

\begin{abstract}
An absolute method of calibrating a neutron source by determining the neutron absorption rate in a manganese sulfate bath is described. In this method slightly more than one liter of solution from the bath is irradiated in a strong neutron flux. The absolute activity of manganese in an aliquot of this activated solution is determined with a $4 \pi$ flow proportional counter. Concurrently, one liter of this solution is added to the inactive bath and after stirring, the counting rate is measured with a dip counter. As the number of radioactive manganese nuclei contained in the liter is obtained from the aliquot activity, the dip counter is calibrated for a known manganese activity. Then the bath is irradiated by the source and the dip counting rate at saturation yields the neutron capture rate by the manganese. From a knowledge of the neutron capture cross sections and the manganese sulfate concentration, the fraction of neutrons captured by manganese is determined. As a result, the total neutron capture rate in the bath at saturation is obtained. A value of $Q=1.25_{0} \times 10^{6}$ neutrons per second was obtained for the National Bureau of Standards source (1954). The standard error is \pm 2 percent.
\end{abstract}

\section{Introduction}

As wide discrepancies between international neutron standards [1] ${ }^{2}$ have been revealed by intercomparisons, it was thought advisable to use two different methods for the calibration of the NBS standard neutron source. Both the emission rate of the NBS standard neutron source [2] and the thermal neutron density in a moderator [3] have already been determined by methods in which the key measurement is the absolute counting rate of alpha particles from thin boron films. The purpose of the present work was to develop an independent method having an accuracy equal to or better than that obtained previously. In the method selected the key measurement is the determination of the absolute activity of an aliquot from an activated manganese sulfate solution.

Two previous absolute calibrations of neutron sources have been made elsewhere in which manganese sulfate $(\mathrm{MnSO})_{4}$ solutions were employed to moderate and absorb the emitted neutrons. O'Neal and Sharff Goldhaber [4] used a simple method. The activity of a tank of manganese sulfate solution is measured with a dip counter after irradiation to saturation with a neutron source and thorough stirring of the active solution. Powdered manganese is added to the solution and the mixture again irradiated to saturation. After removal of the powder, the activity of the solution is measured and the absolute activity of the powder determined. The total activity of the powder is equal to $(1-f) Q$, where $f$ is the ratio of the solution counting rates from irradiations with and without the powder, and $Q$ is the emission rate of the source. When the irradiation

\footnotetext{
1 Present address: Westinghouse Electric Corp., APD, Pittsburgh, Pa.
}

2 Figures in brackets indicate the literature references at the end of this paper. is made with the powder present, $f$ is the fraction of the emitted neutrons captured by the solution.

The Swiss standard source has been calibrated in a similar manner [5]. Instead of using manganese powder, the activity of the solution is measured at two different concentrations to determine the ratio of the captures in manganese sulfate to water (hydrogen). A solution of manganese sulfate is activated in an intense neutron flux from the D-D reaction. The activity of the solution is measured, as before, with a dip counter. Part of the solution is precipitated as manganese carbonate and the absolute activity of a dried sample of this powder determined with a layer counter [6]. This measurement calibrates the dip counter arrangement for this concentration in terms of the manganese activity present. Both methods require the determination of the absolute beta activity of a fairly thick layer of powder and the precision in the neutron emission rates claimed is of the order of 8 percent.

In brief, the NBS standard Ra-Be $(\gamma, n)$ source consists of a beryllium sphere, $4 \mathrm{~cm}$ in diameter, at the center of which a one curie capsule of $\mathrm{RaBr}_{2}$ is placed. As only gamma rays can enter the beryllium, the neutron emission rate is quite constant, unlike a $\mathrm{Ra}-\mathrm{Be}$ $(\alpha, n)$ source, which has an initial growth of about 0.5 percent a year.

\section{Experimental Method}

\subsection{Outline of General Method}

In the present method the unknown source is suspended at the center of a cylindrical tank (approximately $1 \mathrm{~m}$ in both diameter and depth) filled with manganese sulfate solution. After irradiation to saturation and thorough stirring, the activity of the solution is measured with a dip counter. Then in a 
later experiment, slightly more than 1 liter of tank solution is irradiated in a strong neutron flux. A small aliquot (usually $0.075 \mathrm{ml}$ ) of this activated solution is pipetted onto a plastic film and its absolute activity determined with a $4 \pi$ counter. One liter of this solution is added to the inactive solution in the tank, the mixture stirred, and the activity measured with the dip counter. The number of radioactive manganese nuclei initially present in the tank is obtained from the absolute activity of the aliquot after correction for decay to the same arbitrary reference time. Then the ratio of the saturated dip counting rate from the neutron source irradiation to the counting rate for the known manganese activity yields the saturated neutron counting rate in manganese. This counting rate is converted to total neutron capture rate by a factor expressing the ratio of the total capture cross section per millimeter in the bath to the manganese cross section per millimeter. Let

(1) $C_{s}$ be the dip counting rate of the tank solution at saturation from the source irradiation,

(2) $C_{a}$ be the dip counting rate when the liter of activated solution is added to the tank,

(3) $C_{4 \pi}$ be the absolute counting rate of the aliquot of volume $0.075 \mathrm{ml}$.

Then the ratio of the absolute manganese activity in the tank to the dip counting rate when the liter of activated solution is added to the tank and stirred is

$$
R=\frac{1000}{0.075} \times \frac{C_{4 \pi}}{C_{a}}
$$

$R$ may be interpreted as the absolute manganese activity in the tank per unit dip counting rate. The number of neutrons from the source captured per second by the manganese, $Q_{\mathrm{Mn}}$, is

$$
Q_{\mathrm{Mn}}=R C_{s} \text {. }
$$

If all the absorbing nuclei in the tank solution had capture cross sections varying inversely with the neutron velocity, the number of captures per second in the tank, ?, would be

$$
Q=\frac{N_{\mathrm{Mn}}\left(\sigma_{\mathrm{Mn}}+\sigma_{\mathrm{S}}\right)+N_{\mathrm{H}} \sigma_{\mathrm{H}}}{N_{\mathrm{Mn}} \sigma_{\mathrm{Mn}}} Q_{\mathrm{Mn}},
$$

where $N_{\mathrm{Mn}}$ is the number of manganese (and sulfur) nuclei per millimeter $N_{\mathrm{H}}$ is the number of hydrogen nuclei per millimeter; and $\sigma_{\mathrm{Mn}}, \sigma_{\mathrm{S}}$, and $\sigma_{\mathrm{H}}$ are the capture cross sections of manganese, sulfur, and hydrogen for thermal neutrons. Neutron capture in oxygen is negligible for the photoneutron source. Manganese is not a strict $1 / v$ absorber in the epithermal region and a small $(\sim 1 \%)$ correction is given in the appendix. Including the correction term the equation is

$$
Q=\frac{1.012 N_{\mathrm{Mn}} \sigma_{\mathrm{Mn}}+N_{\mathrm{Mn}} \sigma_{\mathrm{S}}+N_{\mathrm{H}} \sigma_{\mathrm{H}}}{1.012 N_{\mathrm{Mn}} \sigma_{\mathrm{Mn}}} R C_{s}
$$

for the concentration used.
A thin-walled Geiger counter, which had proved very reliable, was used as the dip counter.

\subsection{Description of the $4 \pi$ Counter}

A $4 \pi$ flow counter using a 90-percent argon, 10percent carbon dioxide gas mixture was used for the absolute measurement of the specific activity. The counter has two hemicylindrical collecting volumes separated by a plastic film 7.3 by $12.1 \mathrm{~cm}$. Stainlesssteel wires, $0.0025 \mathrm{~cm}$ in diameter and $2.5 \mathrm{~cm}$ above and below the plastic film, extend parallel to the cylinder axes and are supported by kovar seals projecting through the cylinder bases. One-quarter mil mylar films, coated on both sides with 5 to $10 \mu \mathrm{g} / \mathrm{cm}^{2}$ of aluminum, were mounted on a slide, which slipped into position between the hemicylinders (fig. 1).

Pulses from the collecting wires were separately fed by cathode followers to Chase-Higinbotham nonoverloading amplifiers operating at a gain of approximately 30,000. The amplified pulses from each half of the chamber and the coincidence counting rate were recorded with a coincidence circuit having a resolving-time gate width of $2 \mu \mathrm{sec}$. Several tests were made of the performance of the counter. An activated gold foil, $1 \mathrm{~cm}^{2}$ in area, was used to measure the relative counting efficiencies at different positions on the mylar support. When the source was confined to a central region with boundaries $2.5 \mathrm{~cm}$ from the bases and $0.8 \mathrm{~cm}$ from the walls of the hemicylinders, the measured activities, corrected for decay, agreed within the standard deviation of 0.3 percent. A calibrated $\mathrm{Na}^{22}$ source (supplied by $\mathrm{H}$. Seliger of the NBS Radioactivity Section) was measured with this $4 \pi$ counter. The counting rate obtained, when corrected for decay and absorption in the mylar, was in agreement (within the experimental errors) with the original calibration.

The counting rate as a function of collecting wire voltage and counting rate as a function of (integral) discriminator voltage characteristics of the chamber were investigated with neutron irradiated manganese foils. A collecting voltage of 1,950 v was selected in the center of a voltage plateau of more than $200 \mathrm{v}$.

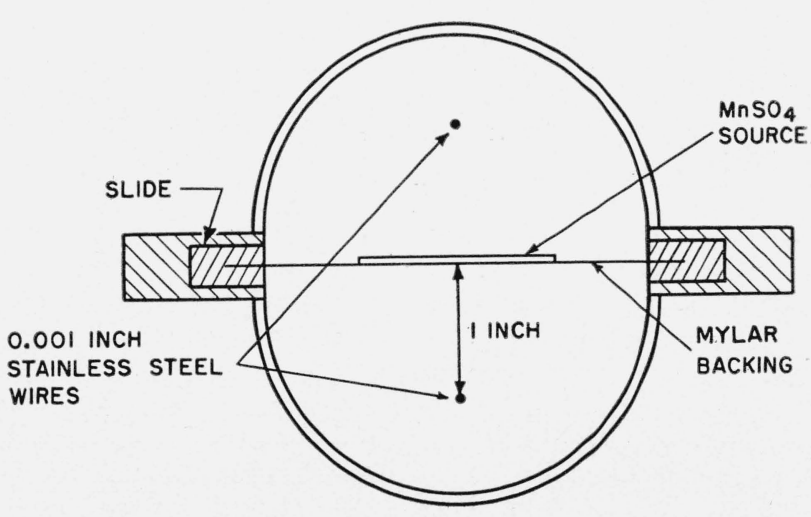

Figure 1. Four $\pi$ counter. 
The decay of the manganese foils was followed for several half-lives on six separate occasions. These measurements yielded a value of $223.7 \pm 0.2 \mathrm{~min}$ for the mean life, which is in good agreement with published values [7].

The $4 \pi$ counting rate is equal to the sum of the individual counting rates from each half of the chamber minus the coincidence counting rate. Usually the collecting wires are connected together so that the $4 \pi$ counting rate is obtained directly; however, knowledge of the individual counting rates gives more information regarding improper operation of the chamber. If a source is not thoroughly dried or the chamber not thoroughly flushed, the trouble is usually quickly detected by observation of the individual counting rates.

\subsection{Preparation of $4 \pi$ Sources}

Considerable difficulty was initially experienced in preparing $4 \pi$ sources, which, after drying, would adhere well to the mylar backing without flaking. The following technique proved the most satisfactory. An area $25 \mathrm{~cm}^{2}$ in extent was wet with a water solution containing 1.5 percent of polyvinyl alcohol and 0.2 percent of triton $\mathrm{X}-100$. Triton $\mathrm{X}-100$ is a potent spreading and wetting agent and the polyvinyl alcohol an excellent binding material. An ultramicroburet, accurate to 1 part in a thousand, delivered $0.075 \mathrm{ml}$ of the activated manganese sulfate solution onto the prepared area. The slide containing the manganese sulfate and a beaker of ammonium hydroxide were placed under a bell jar for a few minutes until the manganese was precipitated. Then the beaker was removed and the bell jar evacuated through a cold trap to dry the film. The mass of one of these sources, determined by weighing, was 25.6 $\mathrm{mg}$, giving a value of about $1.0 \mathrm{mg} / \mathrm{cm}^{2}$ for the average source thickness.

\section{Experimental Data}

Five measurements were taken of the absolute counting rate of the $0.075-\mathrm{ml}$ aliquots with a $1 / 4-\mathrm{mil}$ mylar backing, and one measurement was made with the aliquot sandwiched between two coated mylar films. For the backing alone a typical set of measurements, corrected for decay, was as follows:

(1) Single-chamber counting rate $\sim 2,000$ counts per minute

(2) Coincidence counting rate $\sim 500$ counts per minute. The top side of the chamber (facing the uncovered source) had a counting rate of about 6 percent greater than the bottom half. Table 1 gives the pertinent data for these six cases.

Measurements were made of the $4 \pi$ counting rate as a function of mylar absorber thickness. To obtain a statistically significant effect, aluminum-coated sheets of mylar $7.5 \mathrm{mg} / \mathrm{cm}^{2}$ thick were added above and below the manganese sulfate source deposited on 1/4-mil mylar film. The absorption data are shown in figure 2. A $\frac{1 / 4}{4}$-mil $\left(\sim 1 \mathrm{mg} / \mathrm{cm}^{2}\right)$ mylar absorber causes
TABLE 1. Calibration data

\begin{tabular}{|c|c|c|c|c|c|}
\hline Source- & $\begin{array}{c}4 \pi \\
\text { count- } \\
\text { ing rate }\end{array}$ & $\begin{array}{l}\text { Absorp- } \\
\text { tion } \\
\text { correc- } \\
\text { tion }\end{array}$ & $\begin{array}{c}\text { Abso- } \\
\text { lute } \\
\text { count- } \\
\text { ing rate, } \\
C_{4 \pi}\end{array}$ & $\begin{array}{c}\text { Dip } \\
\text { count- } \\
\text { ing rate, } \\
C_{a}\end{array}$ & a $R=\frac{1,000 C_{4 \pi}}{0.075 \times C_{a}}$ \\
\hline $\begin{array}{l}\text { On } 1 / 4 \text {-mil mylar back- } \\
\text { ing } \\
\text { Do } \\
\text { Do } \\
\text { Do } \\
\text { Do } \\
\text { In 1/4-mil mylar sand- } \\
\text { wich. }\end{array}$ & 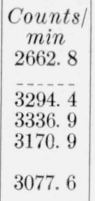 & $\begin{array}{l}1.014 \\
1.014 \\
1.014 \\
1.014 \\
1.014 \\
1.020\end{array}$ & $\begin{array}{c}\text { Counts/ } \\
\text { min } \\
2700.1 \\
3068.6 \\
3340.5 \\
3383.6 \\
3215.3 \\
3139.3\end{array}$ & $\begin{array}{c}\text { Counts/ } \\
\text { min } \\
205.7 \\
237.4 \\
252.6 \\
257.2 \\
246.6 \\
241.7\end{array}$ & $\begin{array}{l}\text { 1. } 750 \times 10^{5} \\
\text { 1. } 724 \\
\text { 1. } 763 \\
\text { 1. } 754 \\
\text { 1. } 738 \\
\text { 1. } 732\end{array}$ \\
\hline \multicolumn{5}{|c|}{ A verage } & 1. $744 \times 10^{5}$ \\
\hline
\end{tabular}

a Each measurement of $R$, not including the error in the absorption factor, has a statistical accuracy of about \pm 0.9 percent, and the error in the average value of $R$ is \pm 0.4 percent. The indicated uncertainties are expressed in terms of the standard error of the mean.

a reduction of $(0.6 \pm 0.1 \%)$ in the counting rate. As the manganese sulfate source thickness is also about $1 \mathrm{mg} / \mathrm{cm}^{2}$, the correction for self-absorption will be of equal size; however, to allow for nonuniformity in the source thickness and variation in the area covered by different sources, the source self-absorption has been taken as $(0.8 \pm 0.4 \%)$. All sources used should have self-absorption corrections falling within these bounds. For the sources deposited on the mylar backing with no cover, the total absorption correction is $(1.4 \pm 0.4 \%)$. At the concentration used and for the cross sections listed in the appendix, eq $3 \mathrm{~b}$ becomes

$$
Q=2.861 R C_{s} \text {. }
$$

Using the average value of $R$ from table 1 ,

$$
?=4.990 \times 10^{5} C_{s} .
$$

With the manganese sulfate bath-dip counter system calibrated in this manner, the absolute calibration of the neutron emission rate from any source is obtained

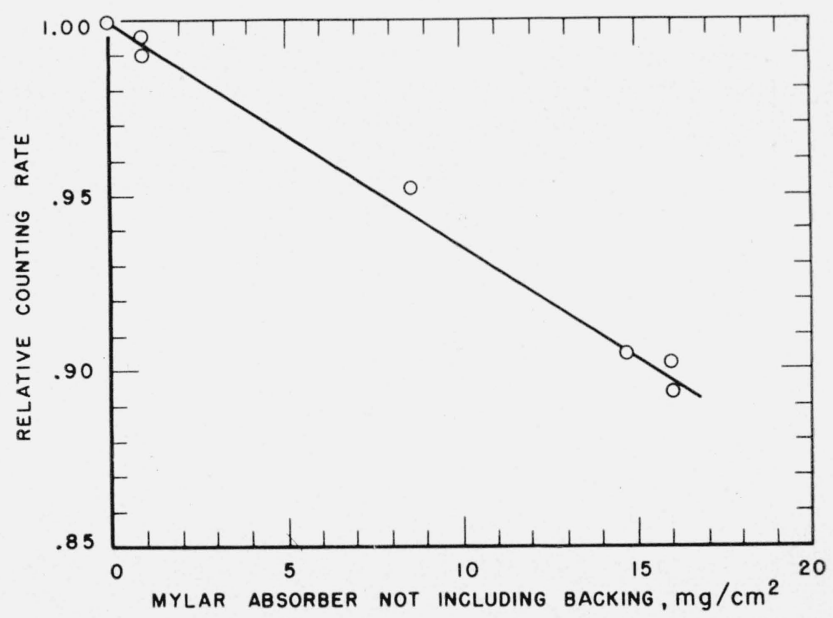

Figure 2. Absorption of $\mathrm{Mn}^{56}$ beta particles in mylar absorber. 
from the dip counting rate at saturation. A small correction must be made for the self-absorption of thermal neutrons by the source when in the bath.

When the NBS standard Ra-Be $(\gamma, n)$ source was placed in the bath, the saturated dip counting rate was $2.49_{8}( \pm 1 \%)$ counts per second, giving a value $Q=1.24_{8} \times 10^{6}$ neutron captures per second in the bath. In pure water the source self-absorption [2] amounted to $6,000 \pm 2,000$ neutrons per second. For the manganese sulfate solution used, the slowing down length is about 4 percent longer than in water, and the mean life of a thermal neutron is only twothirds of the life in water. As a result, the thermal neutron density in the vicinity of the source in the manganese sulfate solution is 60 percent of the value in water, reducing the source self-capture to $3,600 \pm$ 1,500 neutrons per second. Finally, the total emission rate from the source is $\left(1.25_{0} \pm 0.02_{5}\right) \times 10^{6}$ neutrons per second.

\section{Discussion}

The major part of the final standard error in the calibration stems from the uncertainties in the capture cross-section values for manganese and hydrogen, which cause a standard error of 1.2 percent in the capture factor. Recent measurements of the hydrogen cross section by the pulsed neutron decay method yield a value of $0.333 \pm 0.003$ barn [8], compared with the earlier value of $0.321 \pm 0.005$ barn [9]. The value of 0.333 barn, combined with the value of $0.329 \pm 0.004$ barn obtained by Hamermesh, Ringo, and Wexler [10] and $0.332 \pm 0.007$ barn obtained by Harris et al. [11], would give a "best average" of 0.332 barn, compared with the 0.328 barn used. Further work on the decay method is planned by von Dardel in order to resolve some of the uncertainties. However, an increase of 1.2 percent in the hydrogen cross section causes an increase of only 0.8 percent in the value of the neutron emission rate for the concentration of manganese sulfate used.

A value of 13.2 barns was used for the thermalneutron absorption cross section of manganese. As manganese has only one isotope, the activation cross section is equal to the absorption cross section. The activation cross section of manganese was measured [12] by exposing a small cell of manganese sulfate solution in a known thermal neutron flux. After irradiation, the absolute activity of an aliquot of the solution was determined by $4 \pi$ counting (with the same equipment used in the present experiment), and $\sigma_{\text {act }}=13.1_{9} \pm 0.3_{0}$ barns was obtained. In the second supplement of AECU-2040 (neutron crosssection compilation) an absorption cross section of $13.2 \pm 0.4$ barns is listed for the thermal-neutron absorption cross section of manganese and $13.4 \pm 0.3$ barns for the activation cross section (the latter based on a gold absorption cross section of 98 barns). The weighted average of the three values is accurate to $0.1_{9}$ barn.

Actually, systematic errors partially compensate each other, when our own measured value of the manganese activation cross sections is used. Any systematic error in the $4 \pi$ counting technique, which causes an error in the value of $C_{4} \pi$, causes a similar percentage error in the manganese cross-section result. These effects tend to oppose each other, and the net error in the source emission rate is only one-third of the systematic error.

The emission rate of $\left(1.25_{0} \pm 0.02_{5}\right) \times 10^{6}$ neutrons per second obtained in this calibration agrees well with the value of $\left(1.26_{5} \pm 0.03_{7}\right) \times 10^{6}$ neutrons per second obtained by a different method [2]. These two results give a weighted average of $\left(1.25_{5} \pm\right.$ $\left.0.02_{1}\right) \times 10^{6}$ neutrons per second for the emission rate of the standard.

The only American neutron source compared with a foreign source is the Los Alamos source. It was compared with the British standard at Harwell in 1951 by three different methods [13]. As two of these comparisons differed by more than 4 percent, the exact ratio of the source strengths is not precisely known. In an effort to improve this situation, additional international comparisons are planned.

A photoneutron source such as Sb-Be $(\gamma, n)$ could probably be calibrated absolutely to an accuracy within 1 percent by a modification of the present technique. As the gamma-ray energies are less than the photoneutron threshold of deuterium, a bath of manganese sulfate in heavy water could be used. Over 96 percent of the captures would be in manganese, and the error introduced by the cross-section values would be negligible.

The authors express their appreciation to H. A. Bright, NBS Analytical Chemistry Section, for the chemical analyses of manganous sulfate solutions; to H. H. Seliger for the use of his ultramicroburet and Na-22 source; and to R. Tilley and W. Bailey for assistance in performing the experiments.

\section{Appendix. Neutron Capture Above the Cadmium Cutoff Energy}

Manganese is not a strict $1 / v$ neutron absorber in the epithermal region; however, the resonances in the region above the cadmium cutoff energy are principally due to scattering [14], and the epithermal neutron absorption by manganese in solution during the slowing down process is small. Walker has measured the neutron distribution from an Ra-Be $(\alpha, n)$ source in water by means of manganese foils with and without 0.020 -in. cadmium covers [15]. For the space integrals $\left(\mathcal{S}\right.$ activity $\left.\times r^{2} d r\right)$ Walker's measurements give a ratio of $(1.5 \pm 0.1 \%)$ for the integral with cadmium covers to that with bare foils. A small $\mathrm{BF}_{3}$ chamber gave a value of 0.71 percent for the same ratio. As both boron and hydrogen are good $1 / v$ absorbers, 0.71 percent of the neutrons from the source are captured by the hydrogen in water above the cadmium cutoff energy. Manganese, therefore, has about twice the epicadmium absorption of a $1 / v$ detector. 
The resonance escape integral, $P_{r}$, may be used to evaluate the epicadmium captures.

$$
P_{r}=\exp -\int_{E_{\mathrm{Cd}}}^{E_{0}} \sum \sigma_{a i} C_{i} \frac{d E}{\sum \sigma_{s i} C_{i}} \frac{1 E}{\xi E}
$$

where $P_{r}$ is the probability of a neutron escaping capture in slowing down from initial energy $E_{0}$ to the cadmium cutoff energy, $E_{\mathrm{Cd}} ; C_{i}, \sigma_{a i}$ and $\sigma_{s i}$ are the concentration, absorption cross section, and scattering cross section, respectively of the $i$ th element in the medium and $\xi$ is the average logarithmic energy loss per neutron collision. In water and in manganese sulfate solution the slowing down from elastic collisions is almost entirely due to hydrogen, so the equation may be written

$$
P_{r}=\exp -\int_{E_{\mathrm{Cd}}}^{E_{0}} \frac{\sum \sigma_{a i} C_{i}}{\sigma_{s \mathrm{H}} C_{\mathrm{H}}} \frac{d E}{E}
$$

with $\xi=1$ (for hydrogen).

The integral is sensitive to the value used for the cadmium energy (usually taken as 0.3 to $0.5 \mathrm{ev}$ ).

Bothe has derived an expression [16] for the probability of absorption, $\alpha$, of a neutron from an isotropic flux passing through a foil of thickness $d$ and absorption cross section $\sigma$ :

$$
\alpha=1-e^{-n_{\sigma} d}(1-n \sigma d)+(n \sigma d)^{2} E I(-n \sigma d),
$$

where $n$ is the number of nuclei per cubic centimeter. A graph of this function is given in figure 3 for 0.020 in. cadmium; where $\sigma_{a}(E)=\sigma_{t}(E)-7.0$ barns is used for the absorption cross section of cadmium and $\sigma_{t}(E)$, the total cross section, is obtained from AECU-2040. From the graph, $E=0.5$ ev appears to be the best choice for the cadmium cutoff energy. For water, letting

$$
\sigma_{a \mathrm{H}}(E)=\sigma_{a \mathrm{H}}(\mathrm{th})\left[E_{\mathrm{th}} / E\right]^{1 / 2},
$$

and

$$
\begin{gathered}
P_{r}=\exp -\int_{E_{\mathrm{Cd}}}^{E_{0}} \frac{\sigma_{a \mathrm{H}}(\mathrm{th})}{\sigma_{s \mathrm{H}}} \frac{E_{\mathrm{th}}^{1 / 2}}{E^{3 / 2}} d E \\
P_{r}=\exp -\frac{2 \sigma_{a \mathrm{H}}(\mathrm{th}) E_{\mathrm{th}}^{1 / 2}}{\sigma_{s \mathrm{H}}}\left[\frac{1}{E_{\mathrm{Cd}}^{1 / 2}}-\frac{1}{E_{0}^{1 / 2}}\right] .
\end{gathered}
$$

Using $E_{\text {th }}=0.025$ ev, $E_{0} \sim 10^{6}$ ev, $\sigma_{a \mathrm{H}}($ th $)=0.328$ barn, and $\sigma_{s \mathrm{H}}=20.0$ barns, the value of $P_{r}=0.9927$. Thus 0.73 percent of the emitted neutrons are captured by hydrogen in the epicadmium region. This figure is in good agreement with Walker's result for water.

The concentration of the manganese sulfate used in the measurements was $\mathrm{MnSO}_{4}: 1.435$ moles per liter, $\mathrm{H}_{2} \mathrm{O}: 53.33$ moles per liter.

The thermal neutron absorption cross sections used are $\sigma_{a \mathrm{Mn}}(\mathrm{th})=13.2 \pm 0.2$ barns, and $\sigma_{a \mathrm{~S}}(\mathrm{th})=0.49 \pm 0.02$

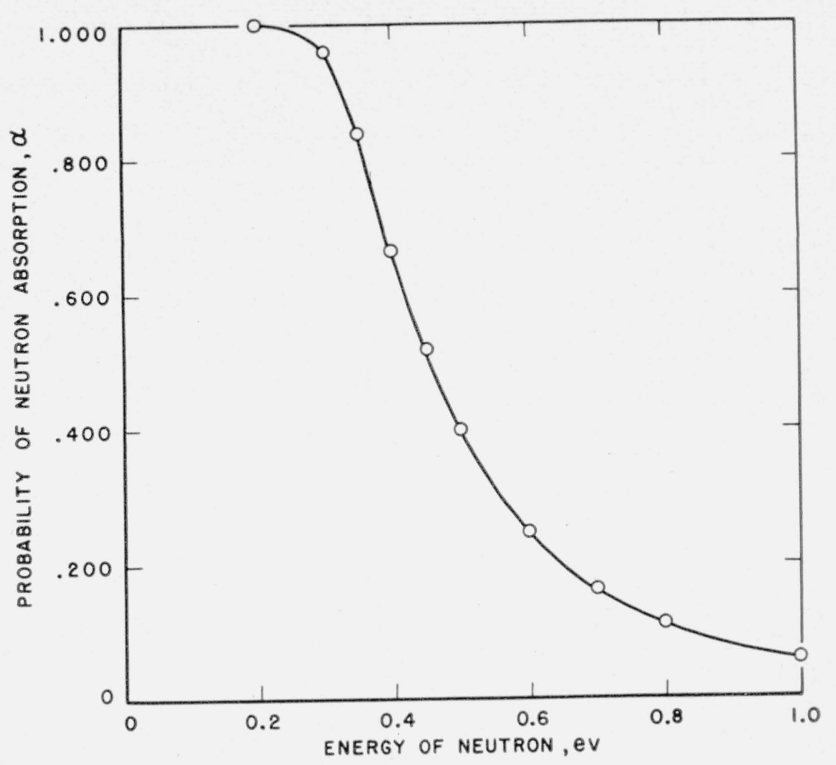

Figure 3. Probability of neutron absorption from an isotropic flux passing through 0.020 in. of cadmium.

barn. For this case, the escape probability is

$$
\begin{gathered}
P_{r}=\exp -\int_{E_{\mathrm{Cd}}}^{E_{0}} \frac{\sigma_{a \mathrm{H}} C_{\mathrm{H}}+\sigma_{a \mathrm{Mn}} C_{\mathrm{Mn}}+\sigma_{a \mathrm{~S}} C_{\mathrm{S}}}{\sigma_{s \mathrm{H}} C_{\mathrm{H}}} \frac{d E}{\xi E}, \\
P_{r}=\left[\exp -\int_{E_{\mathrm{Cd}}}^{E_{0}} \frac{\sigma_{a \mathrm{H}}}{\sigma_{s \mathrm{H}}} \frac{d E}{E}\right][\exp \\
\left.-\int_{E_{\mathrm{Cd}}}^{E_{0}} \frac{\sigma_{a \mathrm{Mn}} C_{\mathrm{Mn}}}{\sigma_{s \mathrm{H}} C_{\mathrm{H}}} \frac{d E}{E}\right]\left[\exp -\int_{E_{\mathrm{Cd}}}^{E_{0}} \frac{\sigma_{a \mathrm{~S}} C_{\mathrm{S}}}{\sigma_{s \mathrm{H}} C_{\mathrm{H}}} \frac{d E}{E}\right] .
\end{gathered}
$$

where $\xi=1$.

Assuming that the thermal-capture cross sections vary as $1 / E^{1 / 2}$, the following values are obtained for the three factors comprising $P_{r}$, respectively,

$$
P_{r}=(0.9927) \quad(0.99604) \quad(0.99985) .
$$

Manganese would capture 0.396 percent of the neutrons from the source in the epicadmium region, if it were a perfect $1 / v$ absorber. From the manganese foil measurements, the manganese in solution will actually capture $(1.5 / 0.72) \times 0.396 \%=0.825 \%$ of the emitted neutrons in the epicadmium region; hence 0.43 percent of the neutrons will undergo resonance captures in the manganese. From the thermal cross section data and the manganese sulfate concentration, 34.68 percent of the remaining neutrons or 34.53 percent of the emitted neutrons are " $1 / v$ " captured by manganese. Finally, (the sum of the $1 / v$ and resonance captures) 34.96 percent of the emitted neutrons are captured by the manganese in the bath. For the concentration used, the manganese captures 1.2 percent more of the emitted neutrons than a perfect $1 / v$ absorber. If this correction were neglected, the resulting error in the emission rate of the source would be 0.8 percent. 


\section{References}

[1] D. J. Hughes, Pile Neutron Research, p. 79 (AddisonWesley Publishers Inc., Cambridge, Mass., 1953).

[2] J. A. De Juren, D. W. Padgett, and L. F. Curtiss, J. Research NBS 55, 63 (1955) RP2605.

[3] J. A. De Juren and H. Rosenwasser, J. Research NBS 52, 93 (1954) RP2477.

[4] R. D. O'Neal and G. Sharff Goldhaber, Phys. Rev. 69. 368 (1946)

[5] F. Alder and P. Huber, Helv. Phys. Acta 22, 368 (1949).

[6] F. Metzger, F. Alder, and P. Huber, Helv. Phys. Acta 21, 278 (1948).

[7] H. H. Hopkins, Jr., Phvs. Rev. 87, 717 (1950); E. Rabinowicz, Proc. Phys. Soc. (London) 63 [A], 1040 (1950); R. Schumann and A. Camilli, Phys. Rev. 84, 158 (1951); L. M. Silver, Phys. Rev. 76, 589 (1949).

[8] G. von Dardel and N. G. Sjostrand, Phys. Rev. 96, 1566 (1954).
[9] G. von Dardel and A. W. Waltner, Phys. Rev. 91, 1284 (1953).

[10] B. Hamermesh, G. Ringo, and S. Wexler, Phys. Rev. 90, 603 (1953).

[11] S. P. Harris et al., Phys. Rev. 91, 125 (1953).

[12] J. A. De Juren and J. Chin, Phys. Rev. 99, 1 (1955).

[13] D. J. Littler, E. E. Lockett, A. D. McRonald, P. W. Mummery, and P. R. Tunnicliffe, British Atomic Energy Research Establishment Report, AERE R/R 776 (1951).

[14] S. P. Harris, C. T. Hibdon, and C. O. Muelhause, Phys. Rev. 80, 1014 (1950).

[15] R. L. Walker, U. S. Atomic Energy Commission Document, MDDC 414 (Oct. 22, 1946).

[16] W. Bothe, Z. Physik 120, 437 (1943).

Washington, June 9, 1955. 